\title{
GROWTH KINEMATICS OF BIVALVE SHELLS
}

UBUKATA, Takao, Geological Institute, University of Tokyo, 7-3-1, Hongo, Bunkyouku, Tokyo, Japan

Microscopic features of bivalve shells in radial and vertical sections were examined both theoretically and empirically. Many of the examined seventy two extant species indicated reclination and curving in the growth directions of aggregated biomineral units as the result of geometric selection. The shape of the growth front formed by the aggregated units determines the profile of internal microgrowth increments. The rate of relative crystal growth generally decreases with the elongation of the structural unit, in association with the curving in the elongation front of the structural units and internal microgrowth increments.

An increment kinematics model based on elementary growth components is introduced for the analysis of bivalve shell growth. The model is expressed by stacking internal microgrowth increments. The shape of the increments is determined by geometric balance of growth components at a given time. The model is empirically well supported by shell biometry. As the results of computer simulation, the increment kinematics model shows a negative feed back system, resulting in conservation of allometric shell growth throughout ontogeny. On the other hand, the overall form of a computer-produced shell is susceptive to the value of parameters, concerning crystal growth under some conditions. These results indicate that the increment kinematics system proposed in this paper inherently possess both ontogenetic stability and inter-individual variability of shell morphology. In such a growth system, the spectrum of bivalve shell convexity is interactively determined by architectural constraints, nature of crystal growth, and anatomical features of the mantle. This model predicts that the biomineralogical nature may play a very important roll not only in constructing the shell microstructure but also in macroscopic shell growth and morphological evolution. 